\title{
Exploring the Transferability of FEATHERS - An Activity Based Travel Demand Model - For Ho Chi Minh City, Vietnam
}

\author{
Hoang Thuy Linh ${ }^{a, b} *$, Muhammad Adnan ${ }^{a}$, Wim Ectors ${ }^{a}$, Bruno Kochan $^{a}$, Tom Bellemens ${ }^{a}$, Vu Anh \\ Tuan $^{c}$ \\ ${ }^{a}$ UHasselt - Universiteit Hasselt, Transportation Research Institute (IMOB), Agoralaan, 3590 Diepenbeek, Belgium \\ ${ }^{b}$ Department of Civil Engineering, University of Transport and Communications, Lang Thoung, Dong Da, Ha Noi, \\ Vietnam \\ ${ }^{c}$ Vietnamese-German Transportation Research Centre, Vietnamese-German University, Bin Duong new city, Vietnam
}

\begin{abstract}
This paper presents descriptive analyses to investigate the transferability of FEATHERS, an activity-based travel demand model developed for Flanders (Belgium), to a region in a developed country to Ho Chi Minh City (Vietnam), a metropolitan area of a developing country, using personal and household travel survey data. The transferability was tested by using indicators which represent individuals' activity and travel behaviors. The results confirm the transferability of the FEATHERS modelling structure to Ho Chi Minh City (HCMC), yet all sub-models require recalibration due to the different cultural settings between the two study areas. The differences in transport mode options, land use patterns and individuals' location choice preferences make transport mode and location choice the least transferable models.
\end{abstract}

Keywords: transferability, activity based travel demand model, action space, schedule pattern

\section{Introduction}

As other developing cities, Ho Chi Minh City, the center for economic growth of Vietnam, is undergoing a strong urbanization process that brings serious congestion and emissions from transportation activities. The city government is seeking tools to effectively manage travel demand through a series of policies/strategies which aim to change the travel behavior of individuals. A travel demand forecasting model based on an individual's participation in activities is an essential tool for this problem. The development of an Activity-Based travel demand Model (ABM) from scratch, however, requires significant resources in terms of time, money and skills while another promising alternative is to use an already existing well-developed ABM system.

Koppelman and Wilmot [1] defined transferability as the “...usefulness of information provided by a model that predicts in a context different from that in which it is estimated". Spatial transferability is the transferability of a model to a different geographical region.

${ }^{*}$ Corresponding author.

E-mail: thuylinh.hoang@uhasselt.be

(C) 2019 International Association for Sharing Knowledge and Sustainability.

DOI: $10.5383 /$ JTTM.01.02.001
Sikder et. al did an extensive review on the transferability studies of ABMs in literature [2]. The authors also proposed a framework with two levels to assess the spatial transferability of ABMs. Case studies are the models in the United States. The first level involves the transferability of the design features of the model system including traveler markets, structure of overall model system, and the spatial and temporal resolution. The second level assesses the transferability of individual models. This framework covers most aspects of an ABM system. However, only the metrics to assess the transferability of individual econometric models have been found. Arentze and Timmerman [3] tested the transferability of the heuristic rule based $\mathrm{ABM}$ model between regions in the Netherlands using some individual and aggregate level measures of the activity schedules. Those studies mostly relate to models transferred between areas in a country that use the same travel dairy design [3] or areas in different countries with similar economic development, social and transportation environment. For more general cases, the foremost task is to investigate if the underlying assumptions used in the base model are applicable in different contexts. There is, however, no empirical study on transferring the assumptions on the decision making mechanism [2]. For cross-culture context, Lee et.al [4] 
summarized several issues including the differences in zoning system, transport system and life-style in a study to transfer FEATHERS [5], an operational ABM model for Flanders [5] (a region in Western country) to Seoul, a region in a developed Asian country. Taken those issues into consideration, this study explores the transferability extent of FEATHERS to HCMC, a metropolitan area of a developing country based on temporal spatial patterns of activity - travel behavior using the travel survey data.

The rest of this paper consists of four parts: the first part reviews some existing ABMs to position FEATHERS with other ABM systems. Following part briefly introduces general information of the two study areas. The third part presents descriptive analysis results and discussions on model transferability. The last part concludes the paper and presents directions for future researches.

\section{Activity-based travel demand models}

Existing ABM systems can be categorized based on the underlying assumptions of the individual decision-making process. They include: econometric models, heuristic rules based models and hybrid models which combine both decision making assumptions in the simulation process. The econometric $\mathrm{ABM}$ was first proposed by Alder and Ben Akiva et.al in 1979 and then Bowman in 1995 [6] developed an operational full-day model. These models assume that individual's daily activity pattern consists of several tours in which activities are scheduled based on a prior assumption of primary and secondary purposes. The next level predicts the tour attributes including transport mode, the destination of the primary activity and tour time of day. The intermediate stop level predicts transport mode, location and time of day for the other activities in tours. All choices in the simulation process are made by discrete choice models which rooted from econometrics.

Arguing that individuals do not always make rational decisions, Arentze and Timmermans [7] introduced ALBATROSS (A Learning Based Transportation Oriented Simulation System) - a fully operational computation process model based on the principle of heuristic search. In ALBATROSS, individual daily activity patterns are generated based on a set of rules, where activities are scheduled and bonded with a skeleton structure of fixed activities. The tours in schedules are then formed in the nature of available time windows between activities. This model also applies the sequential decision process to predict activity's attributes (travel mode, location and time) as in econometric models at tour level and intermediate stop level.

Auld and Mohammadian [8] introduced ADAPTS (Agentbased Dynamic Activity Planning and Travel Scheduling) - a dynamic model in terms of the time dependence of activity planning and scheduling process. It combines heuristics with an econometric model in a discrete event microsimulation. In ADAPTS, the process to generate activities and predict activities' attributes is dependent on the priority of activity planning horizons. However, Auld [9] did not explain the generation and scheduling of full-day activity patterns. Besides, this model was calibrated with a two-week-long GPS(Global Positioning System)-based and activity-travel survey which is unavailable in many regions especially developing countries like Vietnam.

FEATHERS (Forecasting Evolutionary Activity - Travel of Household and their Environmental RepercurssionS) was originally developed by IMOB, Hasselt University, to evaluate transport policy measures for Flanders, Belgium. FEATHERS simulation framework also consists of three levels: daily patterns, tour and intermediate stops, similar to the first two models mentioned above. It, however, combines heuristic rules adapted from ALBATROSS [10] for decisions at daily pattern level and discrete choice models for several models such as transport mode and location choices. With a modular architecture, FEATHERS allows practitioners to customize the system [5] with their preferences around the core scheduling module. The model has been studied for the transferability to the Seoul region of Korea [4, 11] and some other European cities. Those cities are similar in economic development and have well-collected data sources for transport planning purposes.

At this phase of studying the transferability of ABM models for HCMC, we do not consider the transferability of design features of the model system that is the modeling components and the structure, linkage and sequence of all component [2]. Because the modeling framework with a daily pattern, tour and stop levels of either econometric or rule-based models have shown a high possibility to be transferred to many geographical regions where data resource is limited. The model for Jakarta Metropolitan area [12] is an example. The design features of this model is adopted from the framework for Boston and Portland [13]. Regarding individual models, although being criticized for somehow unrealistic representation of daily behavior, but from the practical point of view, discrete choice models allow researchers to represent complicated interactions of individuals' characteristics in scheduling their daily activity and travel routine. To this point, FEATHERS, with the combination of different decision-making mechanisms and the modular architecture, can be considered a promising base application for HCMC.

\section{General information of study areas and data}

\section{sources}

Flanders and HCMC intuitionally seem different in many aspects: economic development, social and cultures, climate and geographical characteristics, transportation infrastructure and so on. Flanders, with a surface area of 13,522 (km2), a population of $6,444,127$ (in 2015), is a geographic region in Belgium divided into five provinces with land use patterns spreading over the region [14]. HCMC, with a total area of $2,061 \mathrm{~km} 2$ and population of $8,127,900$ (in 2015) meanwhile is a metropolitan area in the South of Vietnam with monocentric structure region. The population, as well as the road network, is mainly concentrated in the city center.

FEATHERS was trained on OVG data which is based on the large-scale person travel survey conducted in all Flanders region funded by the Ministry of Mobility and Infrastructure, Flanders, Belgium. Data with similar administrative and geographical level of HCMC is not available for this study. For HCMC, we use the large-scale household travel survey which is known as "Data Collection Survey on Railways in Major Cities in Vietnam" (METROS Study - funded by JICA: the Japan International Cooperation Agency). This survey was conducted from January to April 2014 for HCMC and some parts of the surrounding provinces. Therefore, the trip survey in METROS was filtered for only individuals living and making trips within HCMC. This data covers a similar period as OVG3 in Flanders (being conducted from 2007 to 2013 in phases). Both travel dairy datasets were converted into one-day schedule format and checked for validity. After those steps, there are 1,208 observed schedules of 46,197 individuals in 
METROS; 1,115 observed schedules of 5,879 individuals in OVG3.

Bearing in mind the differences between two study areas, the attributes of household and person described in the two survey questionnaires are defined differently except for generic attributes such as gender and age but FEATHERS currently does not model individuals younger than 18 years old. Most activity types, however, are found similar except "To eat (not at home)". "Visiting someone" and "Recreation, sport, culture" are categorized as a single activity in METROS (see Table 1). The next section presents the comparisons of activity-travel behavior indicators based on three modelling levels in simulation framework of FEATHERS as introduced in the previous section.

\section{Investigating Activity and travel behvaiors of residents in Flanders and HCMC}

\subsection{Analysis Framework}

Manheim [15] pointed out the three levels of human daily choices including activity participation, location to perform those activities and travel between locations. Activity and travel related behavior of individuals is the outcome of the interactions between individuals' preferences and living environments within time and space constraints. Our exploration framework is, therefore, based on the temporal spatial patterns of activity and travel behavior represented by observed schedules, accessibility and mobility of individuals. They are also the main data to build an activity based travel demand model [16]. Arentze and Timmerman in [3, 16] used length of schedules, activity engagement pattern in different time episodes, number of tours to analyze activity schedule patterns in order to develop ALBATROSS and study the transferability of this model. Mobility and accessibility patterns are associated with the observed transport mode and location that individual choose to achieve their activities.

Based on FEATHERS's three-level modeling structure, the indicators to compare activity - travel behaviors in the two study areas to be analyzed are described as below:

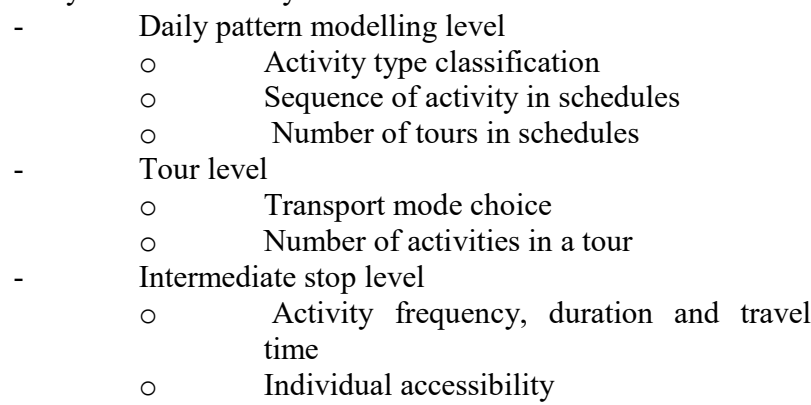

In the next parts, above mentioned indicators are presented.

\subsection{Daily Pattern level}

The main contents of this section summarize the analysis of temporal patterns of activity - travel behaviors with activity type classification, structure of daily activity schedule patterns and the number of home-based tours in the schedules.

The schedule pattern in this study refers to the sequence of activities including in-home (composing of all activities performing at home) and out-of-home activities in a daily routine of individuals (Table 2). A day-long activity schedule in FEATHERS is constructed from a priority assumption based on the activity type and its duration. The primary activities that have higher temporal predictability than others (secondary activities) will be scheduled first into HB tours. The secondary activities will then be added to those tours as the intermediate stops. In order to investigate if this activity classification assumption is similar between HCMC and Flanders, the temporal patterns of each activity could be used as criteria to compare the importance of that activity to the individuals in the two datasets. An optimized classification method developed by Ectors et.al [17] was applied to get the optimal sets of activities for both METROS and OVG3. This method tries to optimally classify different activities into groups - ATC (Activity Type Classes) - with most similar temporal (activity start time and duration) patterns. Fig. 1 visualizes the activity start time kernel density for both datasets as ungrouped and grouped. The activity with high peaks on its probability density curve is more distinctive which mean its start time is easier to be predicted than other activities. "Work"/"To work", "Business trip"/“Company business" (work-related activities) and "Education"/"To school" activities are recognized to have distinctive temporal patterns in both OVG3 and METROS. These activities can be considered as primary activities in both datasets. "To eat-out" activity in METROS shows similar temporal patterns as "Private matters", "Shopping/Market" and "Social/recreation/religious" categories, therefore, it can be considered as a flexible activity which denoted as letter "l". "Others" and "Walk, tour, run" activities in OVG3 and "Pickup/Send off" activity in METROS respectively show more distinctive patterns than the other dataset. So, the scheduling priority for those activities may need revisions in the transferred model.

Table 1. Activity categories in OVG and METROS

\begin{tabular}{|c|c|c|c|}
\hline $\begin{array}{c}\text { In } \\
\text { number }\end{array}$ & OVG3 purpose & METROS purpose & $\begin{array}{c}\text { ATC } \\
\text { symbol }\end{array}$ \\
\hline 1 & Going home & To Home & $\mathrm{h}$ \\
\hline 2 & Work & To Work & $\mathrm{w}$ \\
\hline 3 & Business trip & Company business & c \\
\hline 4 & Shopping & Shopping/Market & 1 \\
\hline 5 & Education & To School & $\mathrm{w}$ \\
\hline 6 & $\begin{array}{l}\text { Visit someone + } \\
\text { Recreation, sport, } \\
\text { culture }\end{array}$ & $\begin{array}{l}\text { Social/recreation/reli } \\
\text { gious }\end{array}$ & 1 \\
\hline 7 & $\begin{array}{l}\text { Services (physician, } \\
\text { bank) }\end{array}$ & $\begin{array}{l}\text { Private matters (e.g. } \\
\text { bank) }\end{array}$ & 1 \\
\hline 8 & Walk, tour, run & Joy riding & $\mathrm{j}$ \\
\hline 9 & $\begin{array}{l}\text { Bring/get } \\
\text { someone/something }\end{array}$ & Pick-up/Send Off & $\mathrm{b}$ \\
\hline 10 & Other & Others & o \\
\hline 11 & & To eat (not at home) & 1 \\
\hline
\end{tabular}

Based on these ATCs, activity categories are re-grouped into 7 ATCs which are symbolized as the "ATC - symbol" column in Table 1 . This step simplifies the day activity schedule in order to compare schedule patterns in two datasets. The observed schedules which are built based on these ATCs form 408 unique schedule patterns in METROS and 976 unique schedule patterns in OVG3. When comparing two datasets, 200 patterns were found common. These patterns share $67.42 \%$ observed schedules in OVG3 and $87.09 \%$ observed schedules in METROS. Table 2 lists ten most frequent scheduled patterns by age and gender. In both datasets, these patterns depend on age stronger than gender. The schedule pattern 7 is found more frequent in METROS than OVG3. The work-based tour is currently not considered in FEATHERS thus it needs to be extended for HCMC model. Assumptions on activity and travel 
behaviors for the less than 18 year-old individuals also need to be added in the transferred model.

Fig. 2 presents the frequency of Home-Based Work (HBW) and Home-Based non-work (HBO) tours of two datasets. In METROS, most frequently observed patterns are $01 \mathrm{HBW}$, $(01 \mathrm{HBW}+01 \mathrm{HBO})$ and $02 \mathrm{HBW}$ tours and these tours are mainly composed of 01 or 02 out-of-home activities. FEATHERS is capable to capture these patterns since its framework allows at maximum 02 work episodes and multiple out of home activity tours.
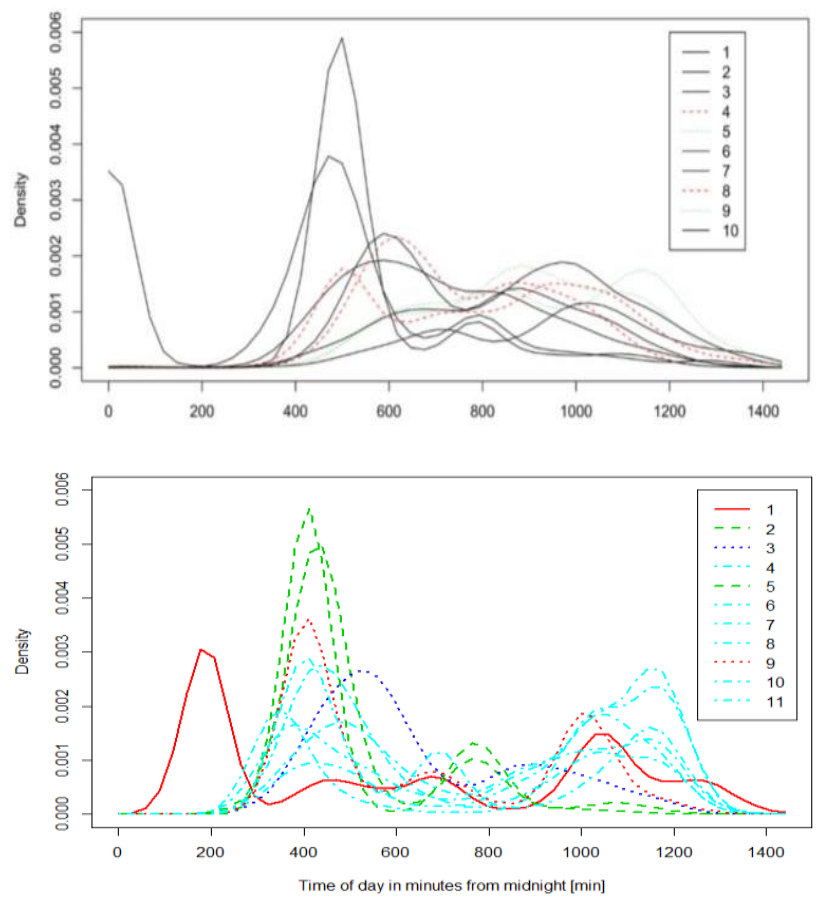

Fig. 1 Kernel density plot activity start time (grouped vs not grouped). Upper: OVG3; Lower: METROS

\subsection{Tour transport mode choice and number of activities}

The mode choice sub-model in FEATHERS only considers the main mode, which is the longest duration mode of the trip. In a home-based tour, there are at least two trips and the tour transport mode of FEATHERS is assumed to be the main mode of the trip from home to work/education or the primary activity (the longest activity duration) in a HBO tour. There are about 9.3\% HB tours in OVG3 and 4.2\% HB tours in METROS that involved different modes (Table 3). The marginal distributions of mode choices over slow mode, private motorized mode and public transport are somehow similar in both datasets. Motorbike (as driver and as passenger) is a unique transport mode in HCMC that used as the main mode for nearly $62 \% \mathrm{HB}$ tours in METROS. This transport mode is popular because of its reasonable cost, higher mobility than bus or car in the peak hours and accessibility in small alleys of HCMC. However, motorbike is highly exposed to the weather and other traffic. Therefore modelling mode choice should include safety and convenience for specific alternative factors [18] additional to vehicle ownership, household context (with or without children), income, travel cost, travel distance, etc. More work should be invested in developing level-of-service data for HCMC in order to capture the sensitivity of mode choice in a different time-of-day due to the different mobility of different transport modes in the mixed traffic in HCMC. As can be seen in Table 3, tours are found more complex in OVG3 than in METROS. Individuals in Flanders seem to prefer combining multiple activities in a home based tour while individuals in HCMC are more likely to perform single activity home based tour. This might be resulted from the different geographical resolution which is described in more details in the latter section.

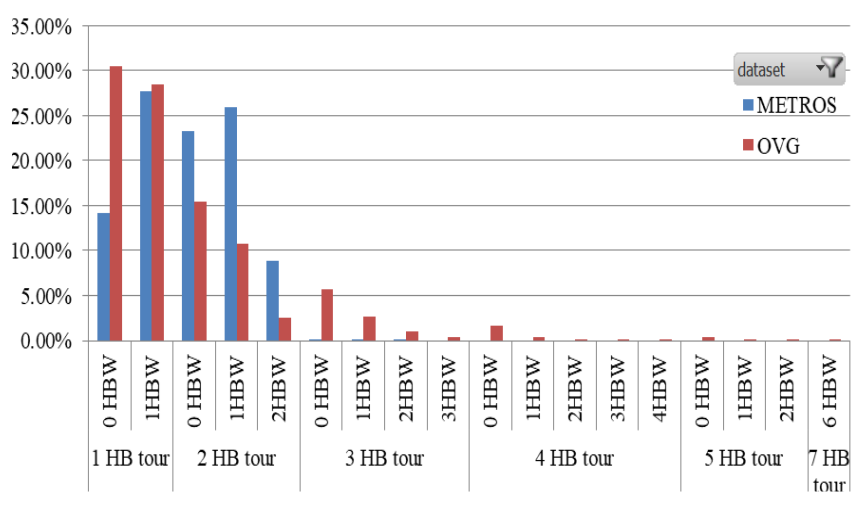

Fig. 2. Frequency of $\mathrm{HB}$ tour and HBW tour

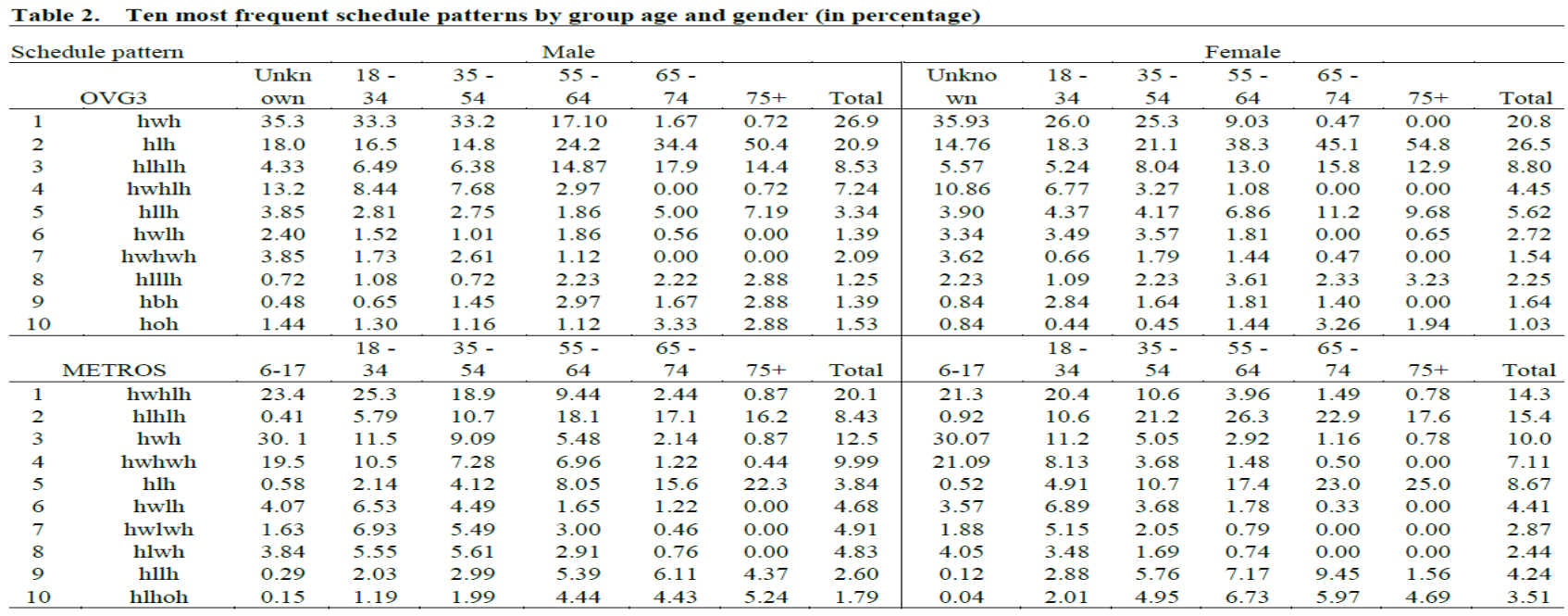


Table 3. Frequency of home based tour by tour mode (in percentage)

\begin{tabular}{|c|c|c|c|c|c|c|c|c|c|c|}
\hline \multirow{2}{*}{\multicolumn{2}{|c|}{$\begin{array}{l}\text { Is single main transport mode used in tour? } \\
\text { Number of activities in tour }\end{array}$}} & \multicolumn{4}{|c|}{ No } & \multicolumn{5}{|c|}{ Yes } \\
\hline & & 1 & 2 & 3 & $4+$ & 1 & 2 & 3 & $4+$ & Grand Total \\
\hline \multirow{5}{*}{$\mathfrak{\jmath}$} & Car driver & 18.28 & 13.96 & 25.41 & 33.57 & 44.91 & 56.21 & 68.53 & 78.18 & 45.94 \\
\hline & Slow mode & 32.26 & 53.96 & 30.81 & 40.56 & 32.48 & 23.49 & 15.73 & 11.82 & 30.94 \\
\hline & Public transport & 26.88 & 18.87 & 24.86 & 11.89 & 6.35 & 1.44 & 1.33 & 0.91 & 6.76 \\
\hline & Car passenger & 22.58 & 13.21 & 18.92 & 13.99 & 16.27 & 18.87 & 14.40 & 9.09 & 16.36 \\
\hline & Number of tours & 186 & 265 & 185 & 143 & 6177 & 975 & 375 & 220 & 8526 \\
\hline \multirow{9}{*}{ 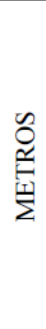 } & Slow modes $=\{$ walk, bicycle $\}$ & 28.14 & 27.45 & 8.94 & 18.06 & 34.96 & 28.68 & 21.50 & 16.55 & 33.21 \\
\hline & Electric bike & 2.71 & 1.57 & 2.16 & 2.78 & 0.70 & 2.01 & 3.22 & 6.90 & 0.99 \\
\hline & Motorbike driver & 17.06 & 14.90 & 65.30 & 55.56 & 50.60 & 62.30 & 70.24 & 73.10 & 51.99 \\
\hline & Motorbike passenger & 34.46 & 21.18 & 11.26 & 11.11 & 10.05 & 5.95 & 4.10 & 4.14 & 9.88 \\
\hline & Car driver & 0.68 & 0.20 & 1.46 & 0.00 & 0.14 & 0.25 & 0.41 & 0.00 & 0.19 \\
\hline & Car passenger & 0.56 & 0.39 & 0.15 & 1.39 & 0.10 & 0.11 & 0.06 & 0.00 & 0.11 \\
\hline & Public transport (taxi, bus, motorbike taxi) & 16.16 & 34.12 & 10.41 & 11.11 & 3.34 & 0.58 & 0.41 & 0.69 & 3.51 \\
\hline & Others & 0.23 & 0.20 & 0.31 & 0.00 & 0.11 & 0.12 & 0.06 & 0.69 & 0.12 \\
\hline & Total number of tours & 885 & 510 & 1297 & 72 & 54595 & 7222 & 1707 & 145 & 66436 \\
\hline
\end{tabular}

model because the travel time variances of most activity types

\subsection{Activity and trip duration}

Table 4 shows statistics of activity and trip duration based on activity type. Regarding activity duration, work/education activities show comparatively smaller variations than other activities in both datasets. This again supports earlier discussion on the distinctive temporal patterns of these activities. Comparing to OVG3, individuals in METROS tend to spend longer duration on work/education activities and shorter duration on leisure ones. This suggests the difference in time use patterns of the people in two areas and confirms the necessary adaptation on activity scheduling priority assumption of FEATHERS. The duration variation of each activity in two datasets is quite similar hence the duration choice sub-model of FEATHERS is possible to capture this behavior of METROS.

The ratio between trip and activity duration are not very different for each activity type between the two datasets at an aggregate level. This suggests the similar preferences of individuals in HCMC and Flanders in the choices of activity location regarding activity duration. However, this similarity does not confirm the transferability of the location choice subare higher in OVG3 than in METROS. Individuals in OVG3 might have more spatially dispersed opportunities for those activity purposes. Taking the "Walk, tour, run" in OVG3 and "Joy riding" in METROS as an example, individuals in HCMC mainly perform this activity within their neighborhood in comparatively short duration compared to Flanders where people travel to specific places for hiking or touring. More details on location choice behavior will be discussed in the next section.

In summary, this descriptive analysis of the day-long activity schedule pattern has identified that there are groups of observed patterns in METROS can be reproduced by FEATHERS simulation structure. The overall daily activity patterns are found largely similar in both datasets. However, there are several aspects that need to be changed in FEATHERS when transferring to HCMC, including: revising the assumptions on scheduling priority for non-work/education activities; adding new sub-model for modelling work-based tour and behavior of less than 18 year-old-individuals; recalibrating all sub-models due to different definitions of most social demographic attributes in the two datasets.

Table 4. Frequency of home based tour by tour mode (in percentage)

\begin{tabular}{|c|c|c|c|c|c|c|c|}
\hline & & Frequency & $\begin{array}{l}\text { Average activity } \\
\text { duration }\end{array}$ & $\begin{array}{l}\text { StdDev of activity } \\
\text { duration }\end{array}$ & $\begin{array}{l}\text { Average of } \\
\text { trip duration }\end{array}$ & $\begin{array}{l}\text { StdDev of trip } \\
\text { duration }\end{array}$ & $\begin{array}{l}\text { Trip-activity } \\
\text { duration ratio }\end{array}$ \\
\hline \multirow{10}{*}{$\stackrel{3}{3}$} & Work & 14.87 & 379.24 & 198.67 & 23.96 & 22.84 & 0.06 \\
\hline & Business trip & 5.90 & 115.69 & 157.93 & 24.19 & 82.82 & 0.21 \\
\hline & Shopping & 21.22 & 43.65 & 78.71 & 14.02 & 23.09 & 0.32 \\
\hline & Education & 6.93 & 315.89 & 165.14 & 19.52 & 20.07 & 0.06 \\
\hline & Visit someone & & & & & & \\
\hline & Recreation, sport, culture & 24.37 & 148.21 & 126.28 & 17.08 & 46.99 & 0.12 \\
\hline & Services (physician, bank) & 5.66 & 51.67 & 76.16 & 13.29 & 13.27 & 0.26 \\
\hline & Walk, tour, run & 3.90 & 147.96 & 177.12 & 56.64 & 88.36 & 0.38 \\
\hline & Bring/get & 11.91 & 32.17 & 92.79 & 13.62 & 13.76 & 0.42 \\
\hline & Other & 5.24 & 176.19 & 185.28 & 14.98 & 57.01 & 0.09 \\
\hline \multirow{10}{*}{ 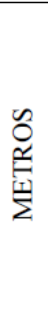 } & To Work & 25.90 & 444.26 & 189.96 & 19.33 & 12.79 & 0.04 \\
\hline & Company business & 0.81 & 130.84 & 162.26 & 20.01 & 13.21 & 0.15 \\
\hline & Shopping/Market & 20.41 & 52.42 & 60.09 & 13.28 & 9.48 & 0.25 \\
\hline & To School & 13.51 & 330.22 & 160.04 & 17.83 & 12.61 & 0.05 \\
\hline & Social/recreation/religious & 5.15 & 92.56 & 71.37 & 15.67 & 10.78 & 0.17 \\
\hline & Private matters (e.g. bank) & 4.53 & 77.82 & 75.99 & 14.73 & 10.47 & 0.19 \\
\hline & Joy riding & 4.12 & 53.43 & 50.29 & 12.15 & 9.23 & 0.23 \\
\hline & Pick-up/Send Off & 4.48 & 52.23 & 121.99 & 13.93 & 9.06 & 0.27 \\
\hline & Others & 6.66 & 95.19 & 113.67 & 16.60 & 14.84 & 0.17 \\
\hline & To eat (not home) & 14.43 & 56.89 & 62.54 & 11.08 & 7.88 & 0.19 \\
\hline
\end{tabular}

\subsection{Analysis of Individual's action space}

The performance of the location choice sub-model in ABM depends on the resolution of land use data and the spatial- temporal effects on activity-travel behavior which can be measured by the space-time accessibility of individuals. The travel survey data in HCMC and Flanders only provided spatial information in sub-zone level with an approximate area of $6 \mathrm{~km} 2$ for both regions. This spatial resolution might absorb 
intra-zonal activities and trips in the compact land use subzones. Since the two study areas differ largely in land use patterns as mentioned in the previous section, it is important to have insights on how this resolution shapes individual's spacetime accessibility when investigating the transferability of the location choice sub-model. The action space (AS) concept is found in literature as a theoretically sound approach to measure space-time accessibility because of the incorporation of constraints, time dimension and the option to use the fixed point locations of fixed activities (home/work) [19]. This study derived the definition by Newsome [20] where AS is an observed potential space that accommodates all potential activity locations that an individual can access within specific time duration (Fig. 3).

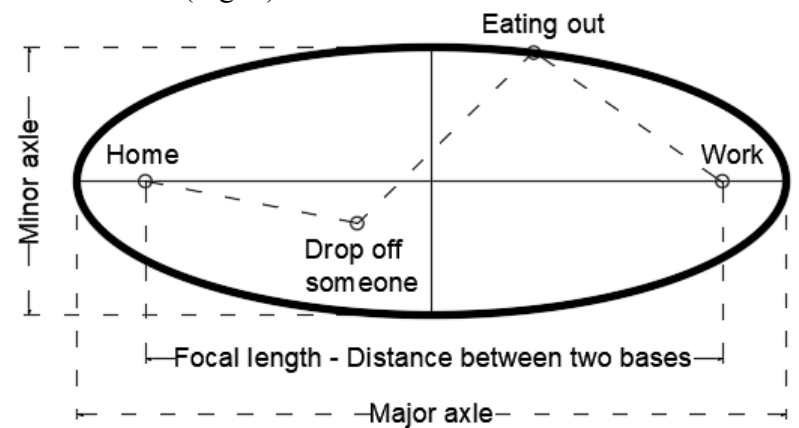

Fig. 3 Trip chaining beginning at 6:30 AM and ending at 8:00AM (Newsome, 1998)

In [21], Dijst used three forms of AS including lines (travel between two bases - no additional stops), circles (only one base) and ellipses (other cases). Our study proposes an additional "point" AS form to capture the action spaces where the bases and intermediate stops are in the same sub-zone. The elliptic action space is constructed using Newsome's method [20] which is based on the distance of two fixed activity locations (bases) such as home location and work location and the furthest activity in the trip chain (Fig. 3). It is difficult to compare the size of potential activity locations between study areas because of the different land use in the two datasets.
Table 5 presents some characteristics of AS such as form, number of out of home activities in an AS, AS size (area or length), and duration of all stops in trip chains, total trip chain duration. One can see that individuals in METROS are more spatially - temporally restricted than OVG3 in terms of AS sizes which are much larger in OVG3 but the ratios between stop duration and total duration are not much different. This large discrepancy is partly explained by limited area and mobility of HCMC compared to Flanders and the different location choice preferences of individuals in the two areas. In METROS, the frequency of the point AS form is comparatively high for both worker and non-worker groups. For that reason, the spatial patterns of activity-travel behaviors in HCMC might be not fully captured with the areal size of the traffic analysis zone. Another indicator discussed in this study is the axle ratio. The value for this parameter is assigned zero (0) for point and line forms, one (1) for a circle and the ratio between the minor axle and major axle for elliptic action space (Fig. 3). Values closer to 1 signify that the activity location is performed away from the bases. Fig. 4 illustrates the spatial - temporal constraints in terms of the axle ratio variation with the starting time of each AS for worker and non-worker in different age groups. In general, there is a time-dependent pattern of workers in both samples. In the time period from $11 \mathrm{AM}$ to $1 \mathrm{PM}$ and 4 $\mathrm{PM}$ to $5 \mathrm{PM}$ in METROS, most workers did not start any activity or performed a work based sub tour with the primary activity closed to workplace. In OVG3, from $12 \mathrm{AM}$ to $1 \mathrm{PM}$ and $4 \mathrm{PM}$ to $5 \mathrm{PM}$ are also the low axle ratio value periods but most people travelled to the locations in different sub-zones other than the base. Individuals in METROS choose out of home activity location more bounded to home or work locations. Due to the differences in location choice preferences and land use attribute data, the location choice sub-models in FEATHERS might not be transferable to HCMC. To this point, this analysis found that the geographic resolution is sensitive to the analysis of location choice behavior and the action space concept can provide descriptive statistics to examine the extent of transferring location choice model between the regions.

Table 5. Action space characteristics by work status

\begin{tabular}{|c|c|c|c|c|c|c|c|c|c|}
\hline & \multirow[b]{2}{*}{ Action space form } & \multicolumn{4}{|c|}{ Worker } & \multicolumn{4}{|c|}{ Non- worker } \\
\hline & & circle & ellipse & line & point & circle & ellipse & line & point \\
\hline \multirow{6}{*}{$\stackrel{\mathscr{S}}{\partial}$} & Avg. nr. of activities in AS & 1.36 & 1.62 & 0.00 & 1.11 & 1.35 & 1.04 & 0.00 & 1.10 \\
\hline & Avg. area $\left(\mathrm{km}^{2}\right)$ or length of line $(\mathrm{km})$ & 2619.77 & 2276.02 & 16.40 & 0.00 & 3161.36 & 980.22 & 7.96 & 0.00 \\
\hline & Avg. duration of stops (minute) & 112.46 & 93.10 & 0.00 & 63.29 & 132.52 & 85.46 & 0.00 & 69.32 \\
\hline & Avg. of total duration (minute) & 151.98 & 142.38 & 21.51 & 74.04 & 177.19 & 128.31 & 14.08 & 81.02 \\
\hline & Stop duration and total duration ratio & 0.74 & 0.65 & 0.00 & 0.85 & 0.75 & 0.67 & 0.00 & 0.86 \\
\hline & Frequency of AS form & $28.53 \%$ & $10.00 \%$ & $53.45 \%$ & $8.02 \%$ & $70.81 \%$ & $0.92 \%$ & $6.67 \%$ & $21.60 \%$ \\
\hline \multirow{6}{*}{ 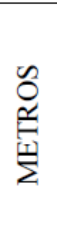 } & Avg. nr. of activities in AS & 1.13 & 1.08 & 0.00 & 1.06 & 1.18 & 1.00 & 0.00 & 1.12 \\
\hline & Avg. area $\left(\mathrm{km}^{2}\right)$ or length of line $(\mathrm{km})$ & 90.13 & 276.87 & 3.31 & 0.00 & 72.25 & 69.45 & 2.77 & 0.00 \\
\hline & Avg. duration of stops (minute) & 97.00 & 61.67 & 0.00 & 60.30 & 94.41 & 110.88 & 0.00 & 57.18 \\
\hline & Avg. of total duration (minute) & 136.61 & 97.42 & 19.85 & 81.33 & 132.96 & 134.38 & 17.72 & 80.32 \\
\hline & Stop duration and total duration ratio & 0.71 & 0.63 & 0.00 & 0.74 & 0.71 & 0.83 & 0.00 & 0.71 \\
\hline & Frequency of AS form & $11.59 \%$ & $4.77 \%$ & $64.36 \%$ & $19.28 \%$ & $31.50 \%$ & $0.05 \%$ & $0.73 \%$ & $67.72 \%$ \\
\hline
\end{tabular}




\section{Flanders: Action space axle ratio with start time and age group - workers}

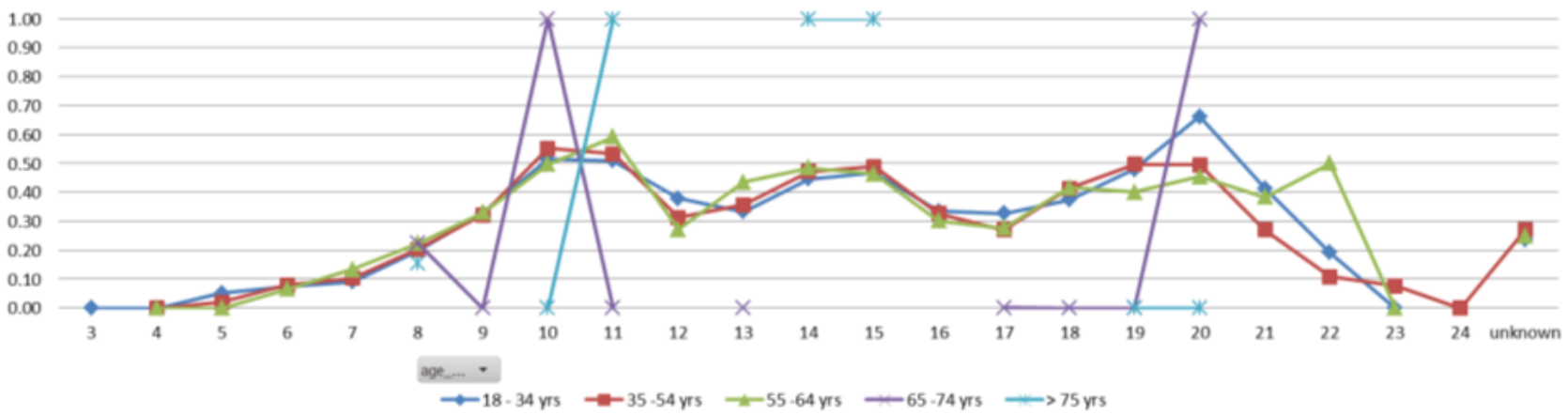

Flanders: Action space axle ratio with start time and age group - Non-workers

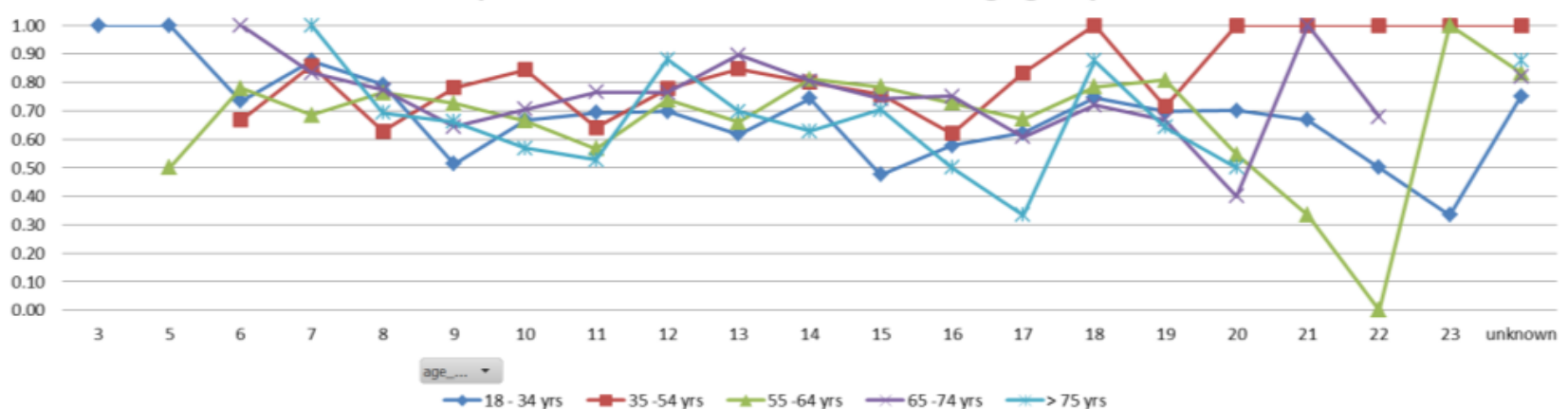

HCMC: Action space axle ratio with start time and age group - Workers

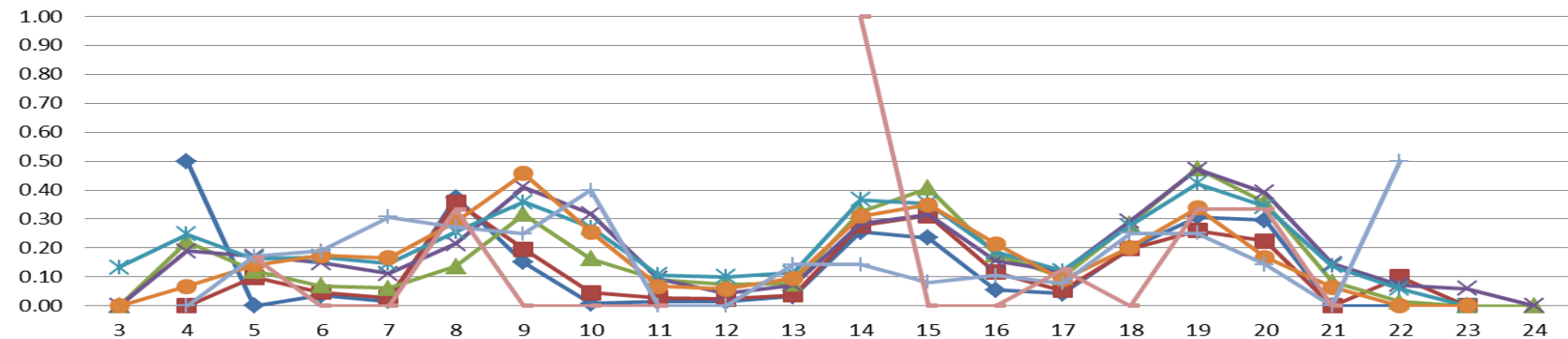
(1)

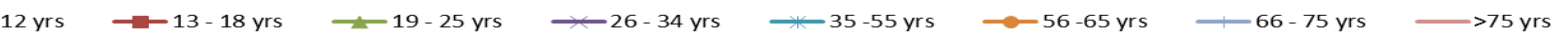

HCMC: Action space axle ratio with start time and age group - Non-workers



\section{Conclusion and Future Research}

This work investigated the (dis-)similarities of activity and travel behavior of Flanders and HCMC in the direction of exploring the extent to transfer FEATHERS to HCMC using the one-day travel survey data. The first task is to assess data inventories in addition to the travel data based on data requirements of the based model. The next task is to analyze the differences in schedule patterns, individual mobility and accessibility which are important in evaluating the transferability of ABM models. The proposed method and 
indicators can effectively describe activity and travel behaviors of individuals in the two areas. The activity schedule pattern in METROS is less complex and among the most frequently observed patterns found in OVG3. Therefore, they can be represented in the scheduling structure framework in FEATHERS without strategical changes. The analysis of individual action spaces also confirms that the location and transport mode choices are the least transferable models.

The future works focus on implementing work-based sub-tour model which mainly composes single travel purposes and depends on workplace land use pattern. Personal and household attributes differently impact activity and travel behavior in both datasets hence all sub-models in FEATHERS have to be recalibrated with HCMC data. The transport mode choice sub-model will be rebuilt with new choice set and specific attributes for motorbikes. Based on existing land use data, the location choice model for HCMC will be constructed in different contexts which are conditional on activity type, individuals' mobility, accessibility and time availability at the tour and stop modeling levels.

\section{Acknowledgments}

This research is financed by the Special Research Fund of Hasselt University. We would also like to thank the JICA study team for their provision the data of METROS project.

\section{References}

[1] Koppelman FS, Wilmot CG. TRANSFERABILITY ANALYSIS OF DISAGGREGATE CHOICE MODELS. Transportation Research Record, https://trid.trb.org/view.aspx ?id=189355 (1982, accessed 5 December 2018)

[2] Sikder S, Pinjari AR, Srinivasan S, et al. Spatial transferability of travel forecasting models: a review and synthesis. International Journal of Advances in Engineering Sciences and Applied Mathematics 2013; 5: 104-128. https://doi.org/10.1007/s12572-013-0090-6

[3] Arentze TA, Timmermans HJP. ALBATROSS $2.0: A$ Learning Based Transportation Oriented Simulation System. Technische Universiteit Eindhoven / EIRASS, https://research.tue.nl/en/publications/albatross-a-learningbased-transportation-oriented-simulation-sys (2005, accessed 1 August 2019).

[4] Lee WD, Joh C-H, Cho S, et al. Issues in Feathers Application in the Seoul Metropolitan Area. Intelligent Transportation and Planning: Breakthroughs in Research and Practice 2018; 979-995.

[5] Bellemans T, Kochan B, Janssens D, et al. Implementation Framework and Development Trajectory of FEATHERS Activity-Based Simulation Platform. Transportation Research Record: Journal of the Transportation Research Board 2010; 2175: 111-119. https://doi.org/10.3141/2175-13

[6] Bowman JL, Bradley M, Shiftan Y, et al. Demonstration Of An Activity-Based Model For Portland, https://trid.trb.org/view/639619 (1999, accessed 11 December 2018).

[7] Arentze T, Hofman F, van Mourik $H$, et al ALBATROSS: Multiagent, Rule-Based Model of Activity Pattern Decisions. Transportation Research Record:
Journal of the Transportation Research Board 2000; 1706: 136-144. https://doi.org/10.3141/1706-16

[8] Auld J, Mohammadian A. Activity planning processes in the Agent-based Dynamic Activity Planning and Travel Scheduling (ADAPTS) model. Transportation Research Part A: Policy and Practice 2012; 46: 1386-1403. https://doi.org/10.1016/i.tra.2012.05.017

[9] Auld J. Agent-based Dynamic Activity Planning and Travel Scheduling Model: Data Collection and Model Development. University of Illinois, 2011.

[10] Bao Q, Kochan B, Shen Y, et al. Activity-Based Travel Demand Modeling Framework FEATHERS: Sensitivity Analysis with Decision Trees. Transportation Research Record: Journal of the Transportation Research Board 2016; 2564: 89-99. https://doi.org/10.3141/2564-10

[11] Hwang JH, Kim H, Cho S, et al. An examination of the accuracy of an activity-based travel simulation against smartcard and navigation device data. Travel Behaviour and Society 2017; 7: 34-42. https://doi.org/10.1016/j.tbs.2017.01.001

[12] Yagi S, Mohammadian A (Kouros). An Activity-Based Microsimulation Model of Travel Demand in the Jakarta Metropolitan Area. Journal of Choice Modelling 2010; 3: 32-57. https://doi.org/10.1016/S1755-5345(13)70028-9

[13] Ben-Akiva ME, Bowman JL. Activity Based Travel Demand Model Systems. In: Marcotte P, Nguyen S (eds) Equilibrium and Advanced Transportation Modelling. Boston, MA: Springer US, pp. 27-46. https://doi.org/10.1007/978-1-4615-5757-9_2

[14] De Vos J. The influence of land use and mobility policy on travel behavior: A comparative case study of Flanders and the Netherlands. Journal of Transport and Land Use 2015; 8: 171. https://doi.org/10.5198/itlu.2015.709

[15] Marvin L. M. The Demand for Transportation. In: Fundamentals of Transportation Systems Analysis, Volume 1. The MIT Press,

[16] Arentze TA, Timmermans HJP. ALBATROSS: A Learning Based Transportation Oriented Simulation System. Technische Universiteit Eindhoven / EIRASS, https://research.tue.nl/en/publications/albatross-a-learningbased-transportation-oriented-simulation-sys $\quad(2000$, accessed 1 August 2019).

[17] Ectors W, Reumers S, Lee WD, et al. Developing an optimised activity type annotation method based on classification accuracy and entropy indices. Transportmetrica A: Transport Science. Epub ahead of print 2017. DOI: 10.1080/23249935.2017.1331275. https://doi.org/10.1080/23249935.2017.1331275

[18] Le TPL, Trinh TA. Encouraging Public Transport Use to Reduce Traffic Congestion and Air Pollutant: A Case Study of Ho Chi Minh City, Vietnam. Procedia Engineering 2016; 142: 236-243. https://doi.org/10.1016/j.proeng.2016.02.037

[19] Kitamura R. An evaluation of activity-based travel analysis. Transportation; 15. Epub ahead of print 1988. DOI: 10.1007/BF00167973. https://doi.org/10.1007/BF00167973

[20] Newsome TH, Walcott WA, Smith PD. Urban activity space: Illustrations and application of a conceptual model for integrating the time and space dimensions. 
Linh et. al. / Journal of Traffic and Transportation Management, 1 (2019) 01-09

Transportation $\quad 1998 ; \quad 25: \quad 357-377$. https://doi.org/10.1023/A:1005082827030

[21] Dijst M. Two-earner families and their action spaces: A case study of two Dutch communities. GeoJournal 1999; 48: 195-206. https://doi.org/10.1023/A:1007031809319 